\title{
sciendo
}

Transport and Telecommunication, 2021, volume 22, no. 2, 196-206

Transport and Telecommunication Institute, Lomonosova 1, Riga, LV-1019, Latvia DOI 10.2478/ttj-2021-0015

\section{ULTRA-WIDEBAND PULSE RADAR WITH DISCRETE STROBOSCOPIC RECEIVER FOR DETECTION OF SMALL TARGETS BEHIND DIELECTRIC OBSTACLES}

\author{
Vladimir Aristov, Gatis Gaigals, Gatis Supols, Eduards Lobanovs, \\ Vents Riekstins and Voldis Zujs
}

\author{
Institute of Electronics and Computer Science \\ Riga, Latvia \\ vladimirs.aristovs@edi.lv
}

\begin{abstract}
Increasing requirements in the field of security, in particular in the transport sector, the rescue work, the inviolability of private property and others urged to research work in the field of radar monitoring people, vehicles or other objects in the environment that not allow make so using the most popular and available technology for production and analysis of video images. These conditions of poor visibility, or even lack thereof, are darkness, bad weather, smoke, dust, wall (roof) buildings and the vehicle body. Existing instruments and special equipment occupy a certain niche in this area, mainly for counter-terrorism operations. However, such equipment is not readily available and extremely high price. In the paper presented research is development of the group's existing radar technology in the field of location through opaque obstacles.
\end{abstract}

Keywords: Ultra-wideband radar; Signal sampling; Step recovery diode; Sub-nanosecond pulses; Radar remote sensing; Radar detection; Radar equipment; Karhunen-Loeve transforms

\section{Introduction}

An increasing number of structures (law enforcement, health care and logistics services, military and researchers) in their everyday work resort to monitoring of various objects, such as: vehicles, people, animals and others. Available video monitoring tools operating both in the visible frequency range and in the infrared are widely used. However, the performance of these tools depend on the visibility of the observable objects: in the cases when observable object is partially or completely behind a covering object (a wall or roof, or a car) or the visibility of the object is degraded by weather conditions (rain, snow, fog, dust storm) or some disaster (fire, landslide, avalanche, earthquake) the monitoring tools based on video analysis can not reach expected level quality of service. In addition, restrictions on the presence of concentrations of people may be tasked definition (with a certain probability) the number of people and their grouping (Choi et al., 2017). This could be at stations, in vehicles and in other public places.

The technology based on the ability of radio waves to freely penetrate non-metallic obstacles can overcome the described difficulties: the radar transmitter emits radio waves that pass through obstacles and reflect from the targets of interest behind obstacles; all the reflections are received by the receiver. Transmitter power is selected based on the requirements of harmlessness effects of radiation on the human body for a long time. Processing the received signals gives a picture (radarogram) of the irradiated space or a special flag of the presence of an object. A number of studies have devoted to this issue, e.g. (Safaai-Jazi et al., 2002; Frazier, 1997; Nguyen et al., 2019). Nevertheless, this direction is open for further research both in the construction of small-sized equipment, and in the creation of processing algorithms that allow get an intuitive result.

This article discusses the prototype of the radar, its components, their uniqueness, the features of the operation of main modules are noted and are shown the capabilities of the radar prototype during monitoring of rooms and hidden objects inside. In order to determine possibilities of the radar the recorded data was processed for the following experiments:

1) scanning the rooms through the wall while radar was moved along the wall;

2) scanning the room with a stationary radar to detect the disappearance of any object behind the wall;

3) scanning through a reinforced concrete floor in order to detect the movement of a person on the lower floor. 


\section{Radar's prototype composition and its characteristics}

\subsection{Technical specification}

The technical characteristics of the radar prototype used in conducting research to detect objects behind dielectric walls are presented in Table 1.

Table 1.

\begin{tabular}{|c|c|}
\hline Parameter & Value \\
\hline Transmitter pulse & $25 \mathrm{~V}$ \\
\hline Maximum input signal frequency of the receiver & $3.5 \mathrm{GHz}$ \\
\hline Transmitter average power & $2 \mathrm{~V}$ \\
\hline Maximum input voltage & $500 \mu \mathrm{V}$ \\
\hline Receiver sensitivity (without data accumulation) & $50 \mu \mathrm{V}$ \\
\hline Receiver sensitivity (with data accumulation) & $114 \mathrm{~dB}$ \\
\hline Radar performance figures & $1.38 \mathrm{MHz}$ \\
\hline Frequency of repetition of probing pulse & $60 \mathrm{~Hz}$ \\
\hline Scan frequency & Bow tie or Vivaldi \\
\hline Antennas & $800 \mathrm{MHz}$ \\
\hline Central frequency of "bow tie" antennas & $1100 \mathrm{MHz}$ \\
\hline Bandwidth of radar with "bow tie" antennas by 0.1 level & $400 \mathrm{MHz}$ \\
\hline Bandwidth of radar with "bow tie" antennas by 0.7 level & $90 \mathrm{~ns}$ \\
\hline Time window (maximum) & $12 \mathrm{bit}$ \\
\hline Sample output & $1024 \mathrm{points}$ \\
\hline Sample count & $1.1 \mathrm{~kg}$ \\
\hline Weight (including antennas and power batteries) & $12 \mathrm{~V} \mathrm{Li-ion}$ \\
\hline
\end{tabular}

\subsection{Receiver and control circuitry}

The block diagram of the receiver combined with the micro-controller and the appearance of the control board are shown in Figure 1. The physical implementation of the receivers blocks (a comparator and a gating pulse shaper and a control circuit with micro-controller) on different boards are chosen in order to minimize the interference to the receiver.

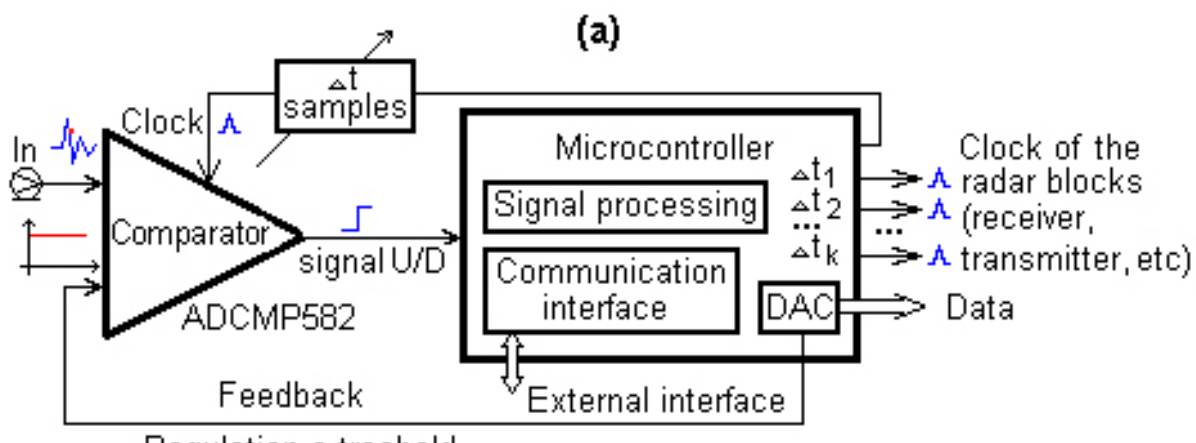

Regulation a treshold (b)

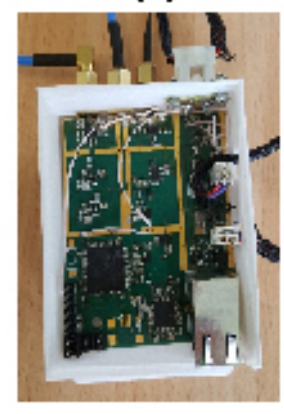

Figure 1. A simplified block-circuit of (a) receiver, combined with the control circuit and appearance of a control circuit (b)

Having a receiver, that allows you to work with input signals up to $5.5 \mathrm{GHz}$, nevertheless, the range 1-2 GHz (determined by the choice of the central frequency of the antennas, at which the ultrawideband antennas bow-tie was used) was chosen for the purposes of sounding through the walls and the reinforced concrete ceiling. This is due to the large attenuation of radio waves in concrete at frequencies more than $2 \mathrm{GHz}$. To record signals of this range, we use digitized low-frequency signals received from a stroboscopic converter. As latter was chosen converter discrete type (DSC). The main element of the DSC 
is a gated comparator, which compares the high-frequency input signal at a specific time moment (after transmitters pulse generation start) with a certain threshold. In dependency to the comparison result the comparator gives a command to increase or decrease the threshold value. With repeated gating at the same time moment of the input signal, the comparator commands of increasing or decreasing the threshold voltage become equally probable. In this case the threshold voltage is assumed to be equal to the input high-frequency voltage at the moment of gating. The moment of gating time is shifted forward and process of comparison series is repeated for the new gating time moment (Hermanis, 2010; Krumins et al., 2009).

The disadvantage of such a discrete converter is the requirement for a high repetition rate of the input signal so that the transformed signal has an acceptable repetition rate, allowing track the movement of objects. However, the industry has mastered the production of ultra-fast and inexpensive comparators. The presence of such a comparator greatly simplifies the circuitry of the converter in comparison to the circuitry of analog stroboscopic converters. In the receiver of the radar prototype a comparator of the ADCMP582 type is used. Extremely minimum duration of the gating interval of 37 ps at 10 ps jitter provides for this comparator equivalent bandwidth $8 \mathrm{GHz}$.

The threshold voltage for the comparator is generated by the microcontroller using its built-in digital-to-analog converter: its output voltage in digital form represents the signal voltage at the gating point. The microcontroller also ensures the formation of clock pulses for the radar units, in particular, the start impulse for transmitted pulse generator and control signals for communication with the external interface. From the attached host computer the microcontroller can be configured to perform some signal preprocessing on-board, for example, a recursive filtering of the signal and its accumulation (discussed in details in 3. Signal processing).

\subsection{Transmitter}

Technically the detection of small targets and targets behind the reinforced concrete walls correspond to detection of targets with small cross section and detection of targets in environments with high signal-path losses. This makes hard to implement requirements to the transmitter: it has to ensure both high amplitude and wide bandwidth of the output signal for excitation of the antenna and radiation of the wideband scanning pulse. The transmitter is based on a two diodes sharpener circuitry with step recovery diode (SRD) that allows to obtain pulses with amplitude of $25 \mathrm{~V}$, a rise time of about 100 ps and a pulse duration at a level of 0.5 of about 200 ps. The operation of SRD diodes based sharpeners and the proper selection of elements are well covered in literature (Pulse and waveform generation with step recovery diodes; Aristov, 2020; Tielert, 1976). Figure 2(a) shows a circuit of this transmitter. The sharpening SRD diodes are D1 and D2. The adjustable charge-pumping current of the diodes is taken from a stable source. The preliminary pulses serving as input for the sharpener on the SRD are formed using avalanche transistor Q1. To reduce the jitter of these pulses, additional filtering of this transistor high voltage power supply is performed. The breadboard of the transmitter with input and output SRM connectors is shown in Figure 2(b). Figures Figure 2(c) and Figure 2(d) show the formed output pulse and its spectral density. In this case the maximum power flux density is not more than $0.2 \mu W / \mathrm{cm}^{2}$ at $1 \mathrm{~m}$ from the antenna (gain of antenna $9 \mathrm{~dB}$ ). This is significantly less than the permissible value adopted in most countries $\left(10-25 \mu \mathrm{W} / \mathrm{cm}^{2}\right)$.

(a)

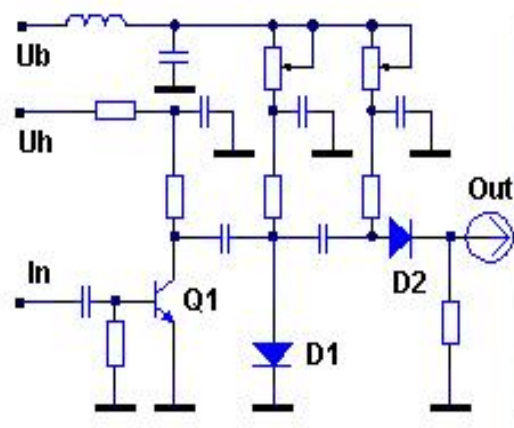

(b)

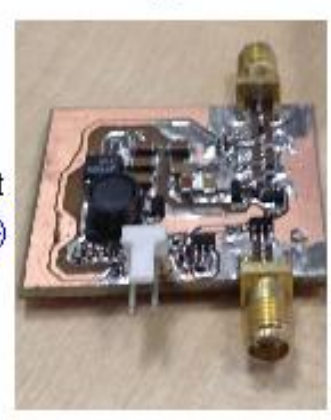

(c)

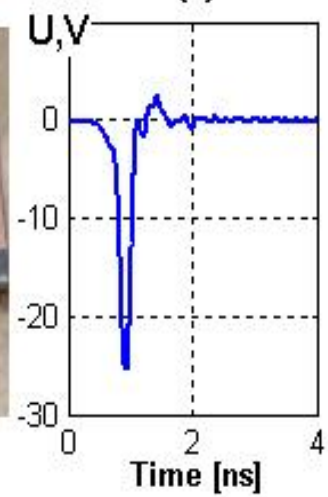

(d)

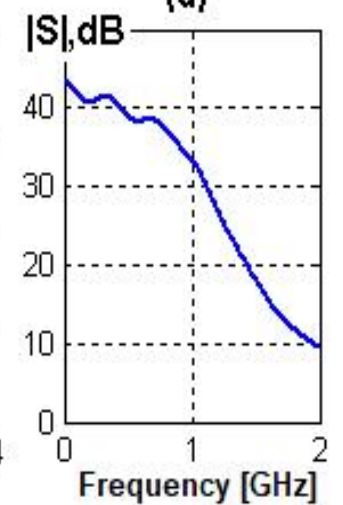

Figure 2. Radars transmitter: a) circuit diagram, b) board, c) output pulse, d) spectral density of the output pulse 


\section{Signal processing}

\subsection{Filtering}

Any signal received during stroboscopic conversion has conversion noise. In addition to it, noise from the transformer of the radar power supply, jitter, external broadcast noise, etc. are superimposed. These noises practically do not affect the processing results if there is a strong signal reflected from the target. However, in the cases of through-wall sounding and detection of small objects, the useful signal approaches the noise level. This gives a false picture of both the presence of the target and its movement. To suppress the above noise, filtering was carried out according to the multi-pass moving average method in a window of length $2 k+1$ using a weighted average. This filter, being similar to the low-pass filter, gives very good results for signals in the time domain (Moving Average Filters). If each continuous signal implementation is represented by a set of discrete samples $x_{i}$, where $i=1,2, \ldots n$, then the moving average filtering algorithm can be represented by the expression:

$$
x_{F i}=\frac{b_{i}}{N_{F}} \sum_{j=0}^{N_{F}-1} x[i+j],
$$

where $N_{F}$ is the averaging window length. The window length and weighting coefficient $b$ were chosen as a compromise between the maximal noise reduction and the minimal distortion of the filtered signal. Signal distortion was evaluated by methods of signal similarity before and after filtering according to the Cauchy-Schwarz formula (Sachs, 2013). A quick analogue of the described filter is the recursive filter (Moving Average Filters), in which the sum value for the next output point is the value of the previous sum minus its starting input point, plus the value of the end input point of the current averaging window. As an example of moving average filtering efficiency the Figure 3(a) shows a signal fragment before (1) and after (2) filtering. The similarity coefficient $K_{k b}$ is 0.985 (for two identical signals it is 1 ). Figure 3(b) shows the spectral density of said signals. It can be seen that the noise reduction is at least $60 \mathrm{~dB}$.

(a)

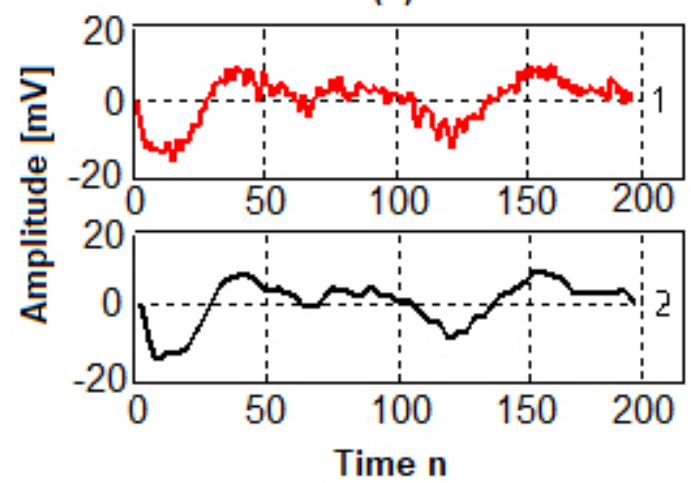

(b)

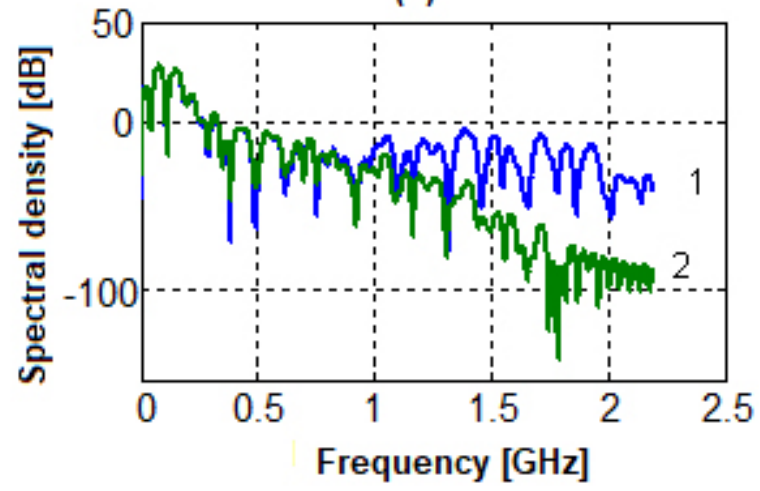

Figure 3. A signal fragment demonstrating noise suppression using the moving average method; 1-signal before filtering, 2-signal after filtering: a) time domain, b) frequency domain

\subsection{Subtraction of a stationary (average) signal}

The duration $T_{S}$ of the signal implementation was selected in order to cover the scanning region with a depth (distance) $L=3-5$ meters. It corresponds to the duration of the signal implementations of $T_{s} \approx 30 \mathrm{~ns}$. However, the useful signal that corresponds to the reflection from the target moving in the research area has a length of the order of several nanoseconds. The rest of the signal include signals from the antenna to the antenna (direct signal) and from stationary objects surrounding the target effectively obfuscate the useful signal. Ultimately making target detection difficult. A similar situation is observed when radar is moved relative to the region of interest. To solve this problem, the average signal is subtracted from the received signal using the following algorithm:

1) When scanning an object of interest during the observation time $T_{o b s}$ an ensemble $\mathrm{A}$ is formed - it is a matrix consisting of $m$ vectors: 
$\mathbf{X}=\left[x_{1}, x_{2}, \ldots x_{n}\right]^{t}$

2) An estimation of the average vector $\bar{X}$ is calculated as:

$\overline{\mathbf{X}}=\frac{1}{m} \sum_{i=1}^{m} \mathbf{X}_{i}$

3) The subtraction of average vector $\overline{\mathbf{X}}$ is performed (Hayashi, 2017) as:

$\mathbf{A}_{w a}=\mathbf{A}-\overline{\mathbf{X}} \mathbf{1}$,

where $\mathbf{1}$ is $m$-sized vector made from ones.

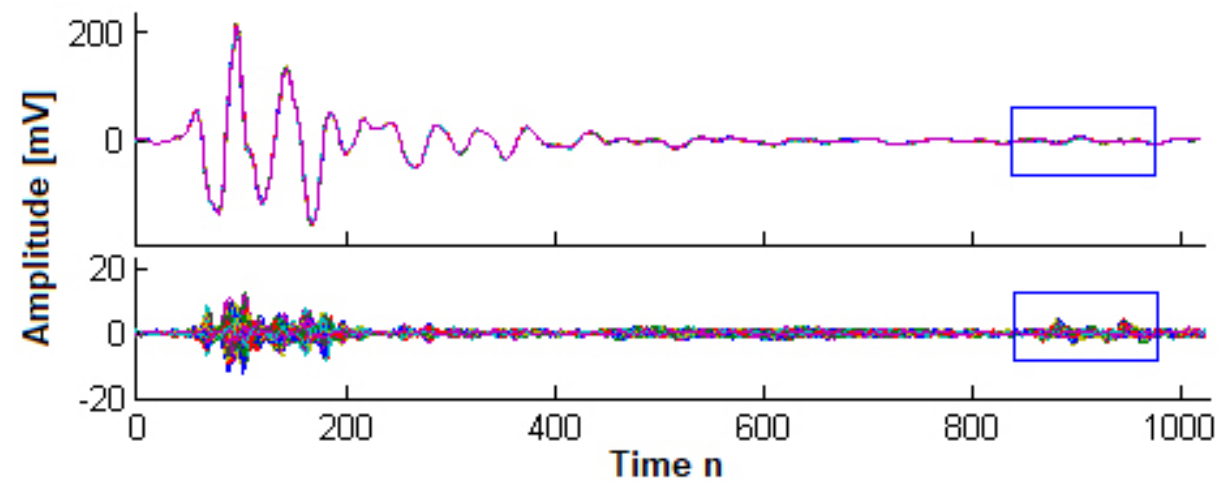

Figure 4. The result of processing the ensemble of signals according to the algorithm "subtraction of the average": above - an ensemble of 796 signal implementations, filtered by a moving average,

below - the same ensemble minus the average. Blue box encloses a reflection from the target

For example, Figure 4 shows the result of processing a real ensemble the conditions for which will be described in the experimental part. In the Figure 4 the zone of the estimated signal from a moving target is surrounded by a rectangle. The figure clearly shows that the ratio of the amplitudes of the signal from the target to the interfering reflections increase by about 10 times due to subtraction of the average signal.

\subsection{Signal redundancy elimination}

In order to get a simple logical answer "yes/no" about the fact if there is a target in the scanning area the redundancy of the signal has to be reduced. In the best case it is necessary to reduce the dimension of the original signal with a value $n$ to an output of one. For this purpose, the Karhunen-Loeve transformation (Limin, 2008; Dony, 2001; Aristov, 2016; Aristov, 2017) was used since this transformation is optimal by the criterion of the minimum mean square error. Moreover, this is achieved with the least number of basic functions that carry out the conversion.

The disadvantages are the complexity of its algorithm, no ready basis functions and the inability to receive the output signal in real time. With the rapid increase in the speed of the embedded computing devices, these shortcomings nullify and the transformation is reduced to a pseudo-real time. The basic functions are unique for each ensemble of observations and are found by the following algorithm:

1) Measuring over time $T_{o b s}$, the ensemble $\mathbf{A}$ is formed (depending on the repetition frequency of the probe pulses and the purpose of the measurement, the time $T_{o b s}$ can vary within very wide limits: from a few milliseconds to several hours);

2) Having the matrix $\mathbf{A}$, we find $\mathbf{A}_{w a}$;

3) Next we find the estimate of the covariance matrix $\mathbf{C}$ :

$\mathbf{C}=\frac{1}{(m-1)} \mathbf{A}_{w a} \mathbf{A}_{w a}^{t}$

4) For the covariance matrix, we find its eigenvalues $\lambda$ and the corresponding eigenvectors $\mathbf{V}$; 
5) The transformation matrix $\mathbf{W}$ consists of the eigenvectors $\mathbf{W}$ :

$\mathbf{W}=\left[\mathbf{V}_{1}, \mathbf{V}_{2}, \ldots \mathbf{V}_{M}\right]$,

where $\mathrm{M}$ is the number of vectors used, $1 \leq M \leq n$. The choice of $\mathrm{M}$ is based on data redundancy, which can be approximately estimated by the ratio of eigenvalues and experimental selection;

6) The final operation is the finding of the transformed signals (images):

$\mathbf{Y}=\mathbf{A}_{w a}^{t} \mathbf{W}$.

In addition to the above mentioned advantages the Karhunen-Loeve transformation has two more advantages:

a) Source signal filtering: in the inverse transformation using only the first, most informative eigenvectors of transformation (main components) that display the largest nonrandom changes in the signal, we get the original signals that are free from minor random changes (Aristov et al., 2014);

b) Unmasking the specific changes of the signal that are hidden under the strong signals: varying used the eigenvectors it can be experimentally possible to uncover changes in the signal that are normally "hidden" behind the strong signals in the unprocessed signal.

\subsection{Correlation processing}

As shown by the results of signal processing the sounding of small dielectric targets, it gives very weak useful signal at a noise level of the receiver. The above processing algorithms in some cases do not give the desired result of detecting such targets.

In order to increase the effectiveness of the target detection the correlation signal processing was used. As previous studies have shown (Karklins et al., 2007; Aristov et al., 2014), the cross-correlation of the explored noisy signals of the observation ensemble with the reference signal can improve the detection of a weak signal. For each implementation in the ensemble $\mathbf{A}$ a selective correlation coefficient $r$ was found with the reference signal $s$ :

$$
r=\frac{\sum_{i=1}^{n}\left(x_{i}-\bar{x}\right)\left(s_{i}-\bar{s}\right)}{\sqrt{\sum_{i=1}^{n}\left(x_{i}-\bar{x}\right)^{2} \sum_{i=1}^{n}\left(s_{i}-\bar{s}\right)^{2}}},
$$

where $\bar{x}, \bar{s}$ - selective average values of the investigated vector signal $x$ and the reference signal $s$. As a reference signal either a model signal corresponding to reflection from a point object or an experimentally selected signal from the ensemble under study was taken.

\section{Experiments and received signals processing results}

\subsection{Scanning a room through a wall while a radar passing along a wall}

A garage with "radio-bright" objects inside, such as radiators (Figure 5), was chosen as the test room. A heating pipe runs along the back wall, several tires (Figure 5(b)) and an assembly table with equipment were laid on the side. The garage door was closed. The radar unit was moved 1 meter from the front wall of the garage along the wall. The travel time was 20 seconds. An ensamble of 473 implementations of 1024 points each were recorded. During processing the filtration, subtraction of the mean and Karhunen-Loeve transformation were used.

Figure 6 shows that filtering and subtraction of the average significantly improves the quality of the picture, allowing identifying hyperbolas, corresponding to the reflections from heating batteries and almost horizontal lines corresponding to the reflections from heating pipes. The characteristic hyperbolas on the upper part of Figure 6(b) correspond to the passage of the radar past the metal gate posts and room corners. Figure 6(c) shows the ensemble radarogram after applying the Karhunen-Loeve transform. For the vectors with the length of 1024 samples the count of principal components is 1024 too. But we took only 50 components starting from $4^{\text {th }}$. It made possible to "highlight" reflections from the pipes and radiators. 
(a)

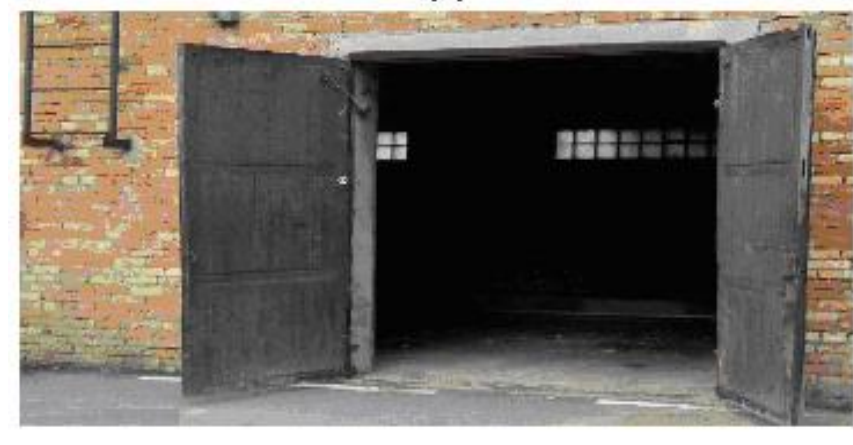

(b)

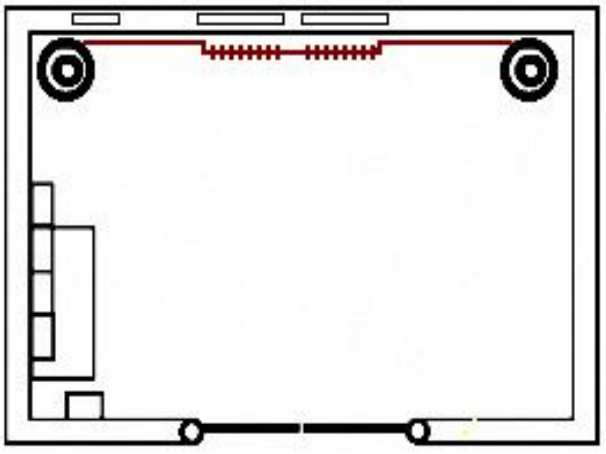

Figure 5. A garage as the scanned room: a) appearance (doors were closed), b) plan of the room

(a)

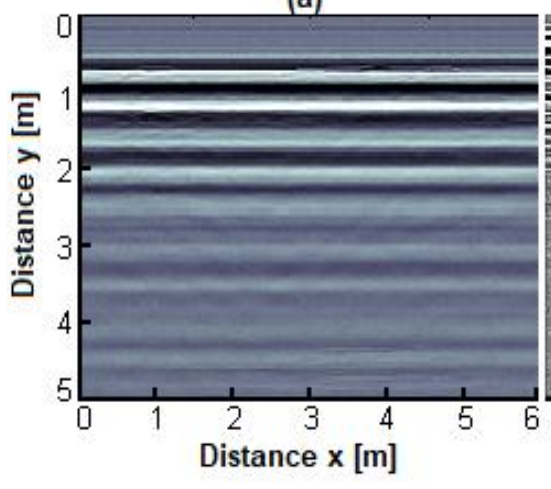

(b)

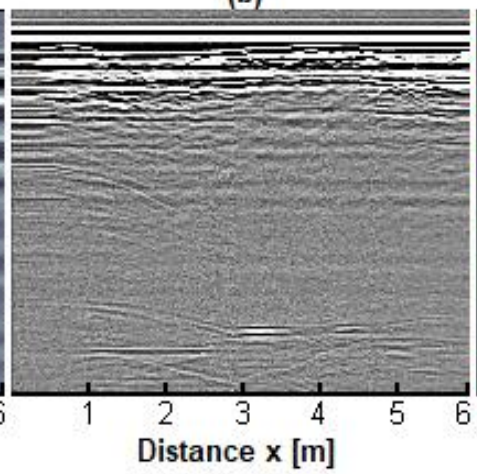

(c)

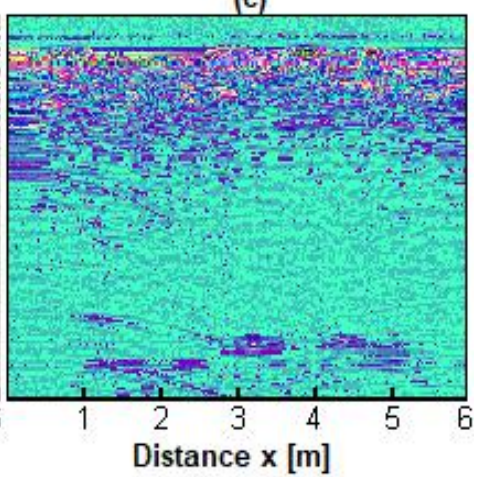

Figure 6. The results (radarogram) of processing the ensemble of signals obtained by scanning the garage: a) raw data; b) the ensemble filtered through a recursive filter and subtracted the average; c) moving average filtering, subtraction of the average and Karhunen-Loeve transformation

\subsection{Scanning through a reinforced concrete floor}

Passing through reinforced concrete floors radio waves experience strong attenuation (Ameri, 2012; Frazier, 1997). So at a frequency $5 \mathrm{GHz}$ the attenuation is $27.2 \mathrm{~dB} / \mathrm{m}$ in both directions. At frequencies less than $2 \mathrm{GHz}$ the attenuation begins to decrease and it becomes moderate.

To study the operation of the radar on scanning objects behind reinforced concrete barriers experiments were carried out with sounding from one floor of a building to another through a reinforced concrete floor. The object of observation was a man. Schematically the scene of this experiment is presented in Figure 7. Radar antennas were located close to the floor. The rise of the antennas led to the decrease of the signal from the object on the lower floor. Up/down vibrations were preliminarily performed with a $50 \times 75 \mathrm{~cm}$ metal sheet in order to "bind" the man's physical location to the location in the signal. The purpose of the experiment was to detect the movement of a man on the lower floor with a metal sheet and without a metal sheet. The distance of man movement on the lower floor was $8 m(4+4)$.

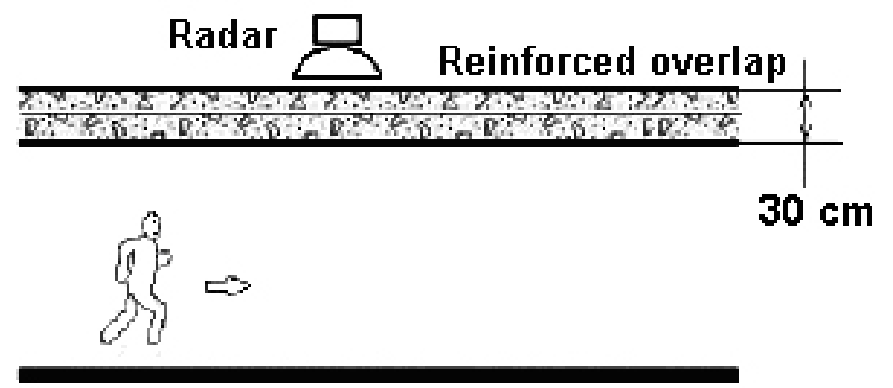

Figure 7. Scene of an experiment with sounding under a concrete floor 
The measurement of each of two experiments was performed for 30 seconds. The results for the case when man carries a metallic sheet and for the case when he does not carry a metal sheet are presented in Figure 8 and Figure 9 respectively. Radarogram analysis gives the following conclusions:

a) On the radarogram without processing the marks of man movement without a metal sheet is not visible;

b) Strong redundancy in signals allows to reduce the dimensionality of the reconstructed signal after the Karhunen-Loeve transformation to several tens of discrete values while maintaining good visibility of man movement with or without a metal sheet;

c) Correlation processing allows you to get a good "fixation" of the passage of a man below the radar.

(a)

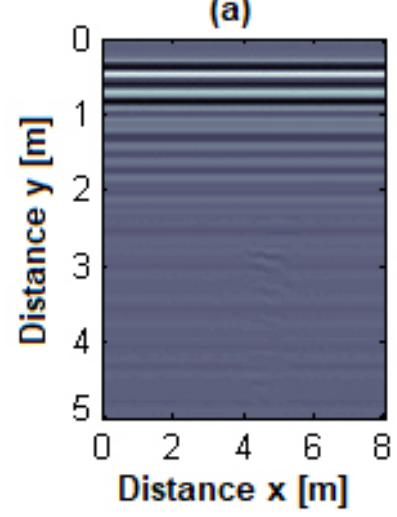

(b)

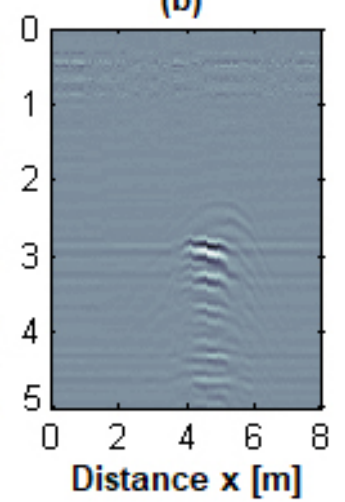

(c)

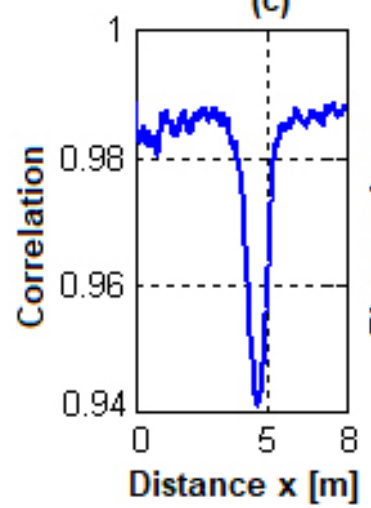

(d)

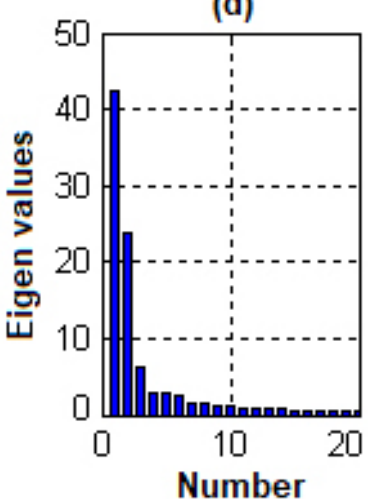

Figure 8. The results of sounding under a concrete floor of a walking man holding a metal sheet at the level of a belt: a) a radarogram of an unprocessed signal; b) a radarogram after processing with a Karhunen-Loeve filter using the first 50 principal components (1024 available); c) correlation coefficient curve; d) the distribution of the eigenvalues of the covariance matrix of the original data ensemble

During analysis of one-dimensional (obtained using only one main component) images of the Karhunen-Loeve transformation it was found that the recovery using the strongest component "blurs" the moment of passage due to the multiplicity of the signal from the parts of the body of the walking man. Using only components with numbers 2 to 4 give a good "fixation" of the passage of a man.

(a)

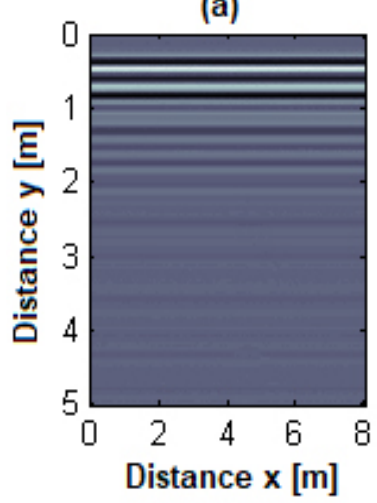

(b)

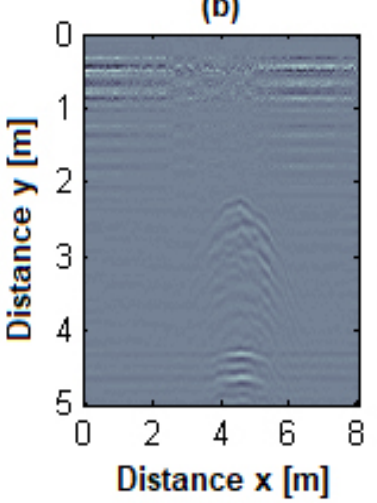

(c)

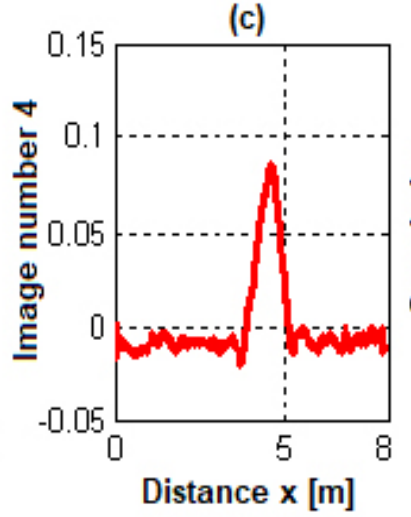

(d)

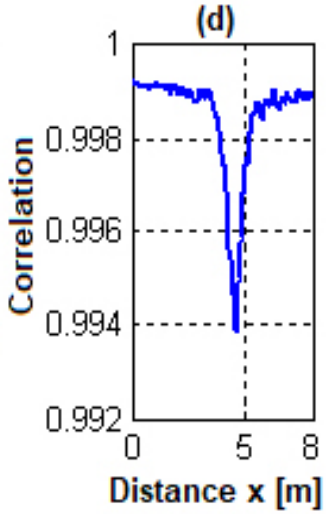

Figure 9. The results of sounding under a concrete floor of a walking man: a) a radarogram of an unprocessed signal; b) a radarogram after processing with a Karhunen-Loeve filter using the first 50 principal components (1024 available); c) curve of the one-dimensional 4th image of the Karhunen-Loeve transform; d) correlation coefficient curve

\subsection{Scanning a room with a stationary radar to detect the disappearance of an object behind the wall}

Layout of the radar is schematically shown in Figure 10. Small targets were placed in the place of red dot. The appearance and dimensions of the small-sized objects participating in the experiment are presented in Figure 11. 
(a)

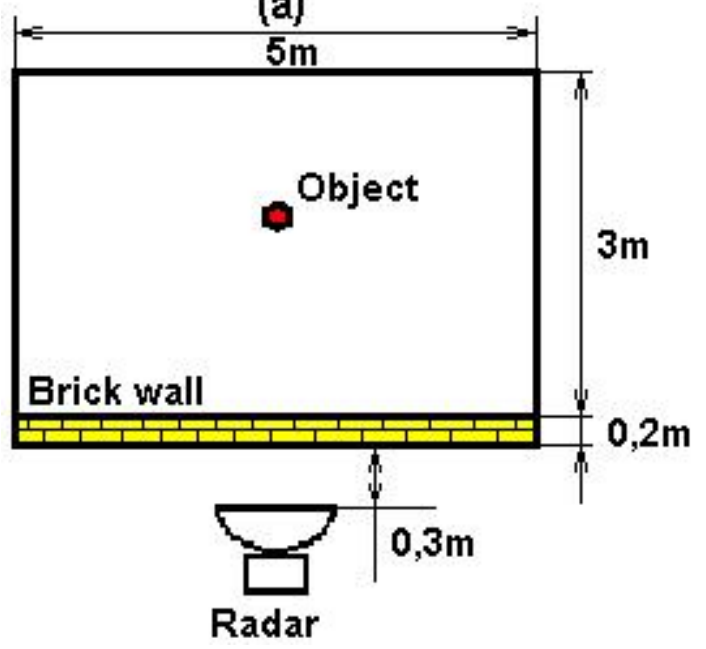

(b)

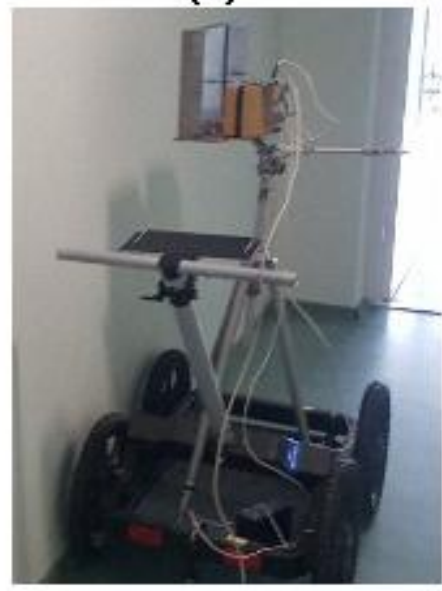

Figure 10. Sounding small objects placed behind a brick wall: a) schematic layout of the experiment; b) a radar prototype

First a room was sounded without a target for 15 seconds. Then the target was placed and sounding without any changes in the radar operation mode, the position of the locator, operators and surrounding objects was carried out. The moment of physical insertion and placement of the object was not entered into the data. After the experiment with the clear table, during following experiments all objects (except man) were placed on the table.

(a)

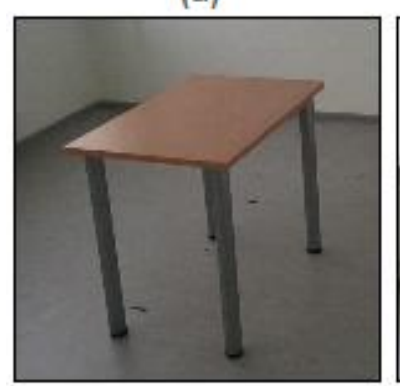

(b)

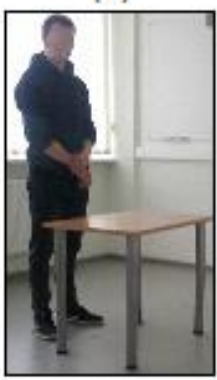

(c)

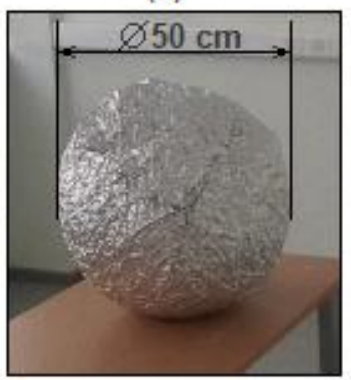

(d)

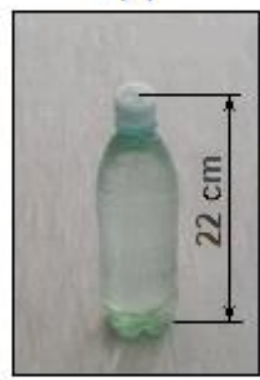

(e)

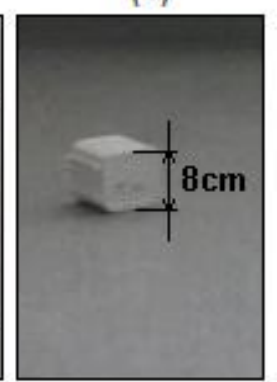

Figure 11. Small-sized objects used in the experiment "there is no object / there is an object": a) a table; b) a man; c) metallized ball; d) a plastic water bottle; e) a metal cube

When analyzing radarograms of unprocessed ensembles only on the radarogram of a man's scan, it was possible to identify a characteristic trace of his appearance indicated by an arrow in Figure 12(b). Figure 12(c) shows a histogram of the distribution of ensemble covariation matrix eigenvalues (see 3.3. Signal redundancy elimination). It analysis shows that the first main component is about 10 times larger than subsequent ones. This indicates a strong redundancy of the received signals. Therefore, the fact of the appearance of an object in the irradiated zone can be fixed only analysing the first (or first two) images of the Karunen-Loev transformation. This is clearly demonstrated in Figure 12(d) where during the observation time $T_{o b s}=30 \mathrm{~s}$ the transition of the first image from one value to another is clearly visible. In the following we used a two-dimensional representation (later referred to as two-dimensional portrait) of the appearance of an object in the sounding zone. This is shown in Figure 13. The values of the first image are plotted on the $\mathrm{X}$ axis, and the second image - on the $\mathrm{Y}$ axis. The analysis of figures Figure 12(c), Figure 12(d) and Figure 13 allows you to draw the following conclusions.

1. The presence of noise (clutter) in the signal during scanning of small objects does not allow to obtain an idealized type of transition from the state "no object (no)" to the state "presence of the object (yes)". In the two-dimensional portrait, this transition would represent a line on the $\mathrm{X}$ axis, i.e., only the first image would change. 
(a)

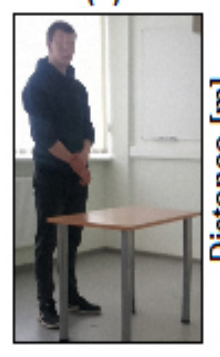

(b)

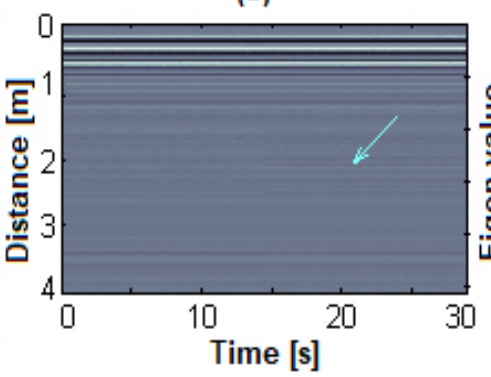

(c)

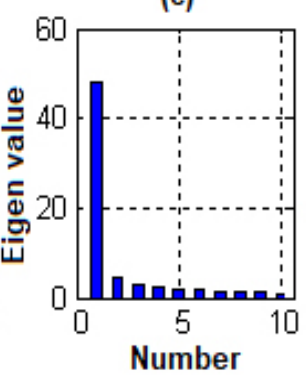

(d)

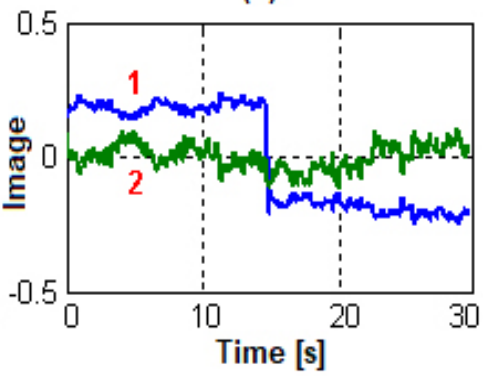

Figure 12. The example of ensembles processing results (in particular, a processing ensemble with a man): a) the experiment setup; b) a radarogram of the raw data (arrow indicates the presence of changes in the signal when the man appears);

c) a histogram of the distribution of the first 10 eigenvalues (1024 available); d) curves of behavior of the first two images

2. The presence of noise in the original signal on a two-dimensional portrait leads to a circular or elliptical appearance of each state (no-yes). If the noise span is approximately the same in both images, then in a two-dimensional portrait the state of the object looks like a circle filled with noise components. The domination of the noise's span in the signal of one of the images, in particular the second image, leads to the "pull" of the circle into an ellipse. The tilt direction of this ellipse is determined by the phase ratio of the predominant interference component in the image's signals. For example, for a metallized ball, these clutter components are anti phase.

3. The transition of the scanned object of their state "no" to the state "yes" looks on a two-dimensional portrait in the form of a connecting two circles (ellipse) a line, corresponding to the transition of the signal of the first image from one level to another. Moreover, by the length of this connecting line can be estimated the radar visibility of the object. The greater the reflectivity of the object is the longer the line of link. For example, of the presented objects, a metallized ball (diameter $50 \mathrm{~cm}$ ) has the greatest radar visibility, and the smallest - a metal cube (with a side size of $8 \mathrm{~cm}$ and with random orientation in relation to the direction of sensing).

(a)

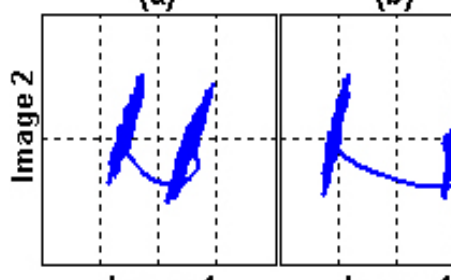

Image 1 (b)

(c)

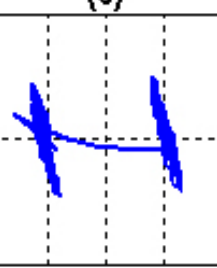

Image 1 (d)

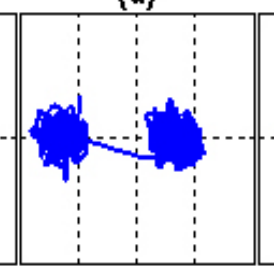

Image 1 (e)

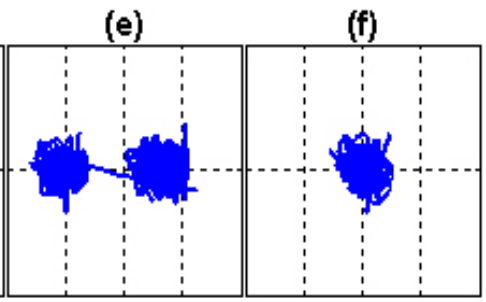

Image 1

Image 1

Figure 13. A two-dimensional representation of the appearance of an object using the first two images of the Karhunen-Loeve transformation; the appearance of the following objects: a) a table; b) a man; c) metallized ball; d) a plastic water bottle; e) a metal cube; f) nothing appears

\section{Conclusion}

Increasing requirements in the field of security, in particular in the transport sector, rescue operations, the inviolability of private housing, etc., are forcing researching work in the field of radar monitoring of the space behind dielectric barriers. In the proposed work, we presented the results of engineering and scientific research on the creation of small-sized ultra-wideband pulse radar and original algorithms for processing signals received using this radar.

Radar performance figures equal to $114 \mathrm{~dB}$ allows to receive a signal from small targets, e.g., from a metal cube with a side of $8 \mathrm{~cm}$ or a man behind a $20 \mathrm{~cm}$ thick brick wall. Scanning the space behind a reinforced concrete floor, that has the highest attenuation compared to other dielectric barriers, made it possible to obtain signals from a moving person.

The proposed processing algorithms for signals received from the radar such as: filtering, subtracting the average, Karhunen-Loeve transform, correlation processing allow us to identify signals, weakened when passing through obstacles on the background of strong masking signals from surrounding objects, by which we can definitely make a conclusion about the movement of the object or its presence. 


\section{Acknowledgment}

This investigation is performed in the framework of the EDI (Institute of Electronics and Computer Science) Project No. 1.1.1.1/18/A/139 co-financed by the ERAF and "Multidimensional Aerial and Facility Scanning System (MAFSS)" in cooperation between the "DATA Group," the University of Latvia's Mathematical and Informatics Institute, and EDI.

The team of authors is grateful to the students Gailis Raivo, Sandis Bondars and Janis Simanovics who actively participated in the experimental work.

\section{References}

1. Choi, J.W., Yim, D.H. and Cho, S.H. (2017) People Counting Based on an IR-UWB Radar Sensor. IEEE SENSORS JOURNAL, 17(17), 5717-5727.

2. Safaai-Jazi, A., Sedki, M. R., Ali, M. and Ahmet, B. (2002) Report on Through-the-Wall Propagation and Material Characterization. Virginia: Virginia Polytechnic Institute and State University. (240610111).

3. Frazier, L.M. (1997) Radar surveillance through solid materials. Command, Control, Communications, and Intelligence Systems for Law Enforcement, 2938. Retrieved: https://doi.org/10.1117/12.266733 (Aug. 14, 2020).

4. Nguyen, T.P., Tang, L., Demir, V., Hasan, S.F., Minh, N.D. and Mukhopadhyay, S. (2019) ReviewMicrowave Radar Sensing Systems for Search and Rescue Purposes. Sensors, 19(13), 2879. Retrieved: https://www.mdpi.com/1424-8220/19/13/2879 (Aug. 14, 2020).

5. Hermanis, E. (2010) Analytic model and bilateral approximation for clocked comparator. IEEE Signals and Electronic Systems, 4, 185-188.

6. Krumins, K., Petersons, V. and Plocins, V. (2009) Features of Implementation of the Modified "upand- down" Method. Electronics and Electrical Engineering, 5(93), 51-54.

7. Pulse and waveform generation with step recovery diodes. Hewlett Packard, Printed in USA, Application note 918, pp. 1-25. Retrieved: http://hpmemoryproject.org/an/pdf/an_918.pdf (Aug. 14, 2020).

8. Aristov, V. (2020) Mathematical Analysis of the Nanosecond Pulse Generator on Two SRD Diodes Used in UWB Radars. Automatic Control and Computer Sciences, 54(3), 1-8.

9. Tielert, R. (1976) Subnanosecond-pulse generator employing 2-stage pulse step sharpener. Electronics letters, 12(3), 84-85.

10. Moving Average Filters. The Scientist and Engineer's Guide to Digital Signal Processing, Chapter 15, 277-284. Retrieved: https://www.analog.com/media/en/technical-documentation/dsp-book/dsp_book_ Ch15.pdf (Aug. 14, 2020).

11. Sachs, J. (2013) Handbook of Ultra-Wideband Short-Range Sensing. Germany: WILEY-VCH Verlag $\mathrm{GmbH} \& \mathrm{Co}$. KGaA.

12. Hayashi, F. (2017) Econometrics. Translation from English. Moscow: Publishing House "Delo" (in Russian).

13. Limin, W. (2008) Karhunen-Loeve Expansions and their Applicotions. Ann Arbor: UMI Diddertation Publishing. UMI Number: U615901.

14. Dony, R.D. (2001) Karhunen-Loeve Transform. Boca Raton: CRC Press LLC.

15. Aristov, V. (2016) Karhunen-Loeve transform as a tool to eliminate signal's redundancy, when small targets detection. Sciences of Europe, 2(2), 53-57 (in Russian).

16. Aristov, V. (2017) Distance localization of the moving object by applying the Karhunen-Loeve transform to ultra-wideband impulse signals. Automatic Control and Computer Sciences, 51(5), 294-300.

17. Aristov, V., Krumins, K. and Plocins, V. (2014) Assay of Concurrent Use of Correlation and Principal Component Analysis for Tasks of UWB Radar. Automatic Control and Computer Sciences, 48(5), 296-302.

18. Karklins, V. and Krumins, K. (2007) Correlation filtering of statistically converted signals. Automatic Control and Computer Sciences, 5, 70-77 (in Russian).

19. Ameri, A. (2012) Long-Range Ultra-Wideband Radar Sensor for Industrial Applications. Germany: Kassel University Press. 